\title{
Caracterización del Ciclo de Desarrollo Embrionario y Larval de Sarda chiliensis chiliensis (Alva, 1987) en un Sistema Acuícola de Recirculación (SAR)
}

\author{
Characterization of the Stages in the Cycle of Embryo Development \\ Sarda chiliensis chiliensis (Alva, 1987) in an Aquatic Re-Circulation System (SAR)
}

Miranda, L..; Vilaxa, A..*; Ávila R.**** \& Rodríguez, M. ${ }^{* * * * *}$

MIRANDA, L.; VILAXA, A.; ÁVILA R. \& RODRÍGUEZ, M. Caracterización del ciclo de desarrollo embrionario y larval de Sarda chiliensis chiliensis (Alva, 1987) en un sistema acuícola de recirculación (SAR). Int. J. Morphol., 32(4):1492-1501, 2014.

RESUMEN: Los sistemas acuícolas de recirculación (SAR) son utilizados cada vez más en la acuicultura, especialmente en el norte de Chile en donde las condiciones ambientales son propicias para el cultivo de especies nativas como el bonito (Sarda chiliensis chiliensis), del cual se desconoce su desarrollo embrionario y larval, lo que complica evaluar la factibilidad técnica y económica de desarrollar su cultivo en SAR. Por ello se determinó las bases para su cultivo a partir de enero de 2013 , durante 45 días. Los periodos embrionarios caracterizados fueron 31 en 71,83 horas, definidos en 5 estadios: Mórula, Blástula, Gástrula, Neurulación y Metamería. Del desarrollo larval se observaron 35 periodos en 519 horas, un estadio prejuvenil observado a las 591 horas y uno juvenil. Los huevos fueron translúcidos de tipo telolecítico de crecimiento lento en relación a Sarda sarda y Sarda ch. lineolata (especies con las que fue comparada), ya que le tomó casi 3 días eclosionar. Las tasas de sobrevivencia y eclosión fueron altas $(86,316 \% \pm 9,808$ y $89,596 \% \pm 11,683$ respectivamente), por esto las condiciones serían propicias para su desarrollo. Las larvas en tanto, presentaron más semejanzas con Sarda sarda, en los primeros periodos de desarrollo. El periodo crucial fue entre las 81 a 89 horas de vida, cuando se reabsorbió la gota oleosa; ésta además fue un carácter diferenciador con las otras 2 especies, ya que fue la única que presentó 1 gota oleosa en el periodo larval. Se infiere la factibilidad de un hatchery de Sarda ch. chilensis, debido a las semejanzas con Sarda sarda, y dado el éxito obtenido de ésta en cultivo. De los caracteres morfométricos se sugiere monitorear en el futuro, los que representan las mayores variabilidades dentro de los estadios de desarrollo, que fueron longitud y alto de cabeza y longitud y alto de ojo.

PALABRAS CLAVE: Desarrollo embrionario; Sarda chiliensis chiliensis.

\section{INTRODUCCIÓN}

La disminución de recursos pesqueros en el norte de Chile, ha obligado a una búsqueda de alternativas de desarrollo, donde la acuicultura de peces marinos silvestres se alza como una posibilidad verdadera, en una zona donde esta actividad no ha sido explotada, siendo las condiciones ambientales propicias para el desarrollo de especies nativas como el bonito (Sarda chiliensis) de gran interés comercial (Vilaxa et al., 2009). La demanda de atunes ha incrementado el esfuerzo de pesca sobre especies como el atún de aleta amarilla Thunnus albacares y la albacora
T. alalunga, ambas se encuentran en peligro de sobreexplotación, por lo que se ha puesto la atención en otros recursos de la misma familia como Sarda ssp. (Yoshida, 1980). El Bonito, es una especie restringida al este del océano Pacífico (Collette \& Chao, 1975), se presenta de forma discontinua desde Chile hasta el Golfo de Alaska (Schultz, 2003), a través de dos subespecies separadas por una población tropical de Sarda orientalis. La subespecie del hemisferio sur, $S$. ch. chiliensis, se presenta desde Máncora, Perú, justo al sur del Golfo de Guaya-

\footnotetext{
* Universidad Tecnológica de Chile, INACAP, Chile.

** Facultad de Ciencias, Departamento de Biología, Centro de Estudios Marinos y Limnológicos, Universidad de Tarapacá, Chile.

*** Facultad de Ciencias, Centro de Estudios Marinos y Limnológicos, Uniuversidad de Tarapacá, Chile.

***** WwW.sampleconsultores.cl
} 
quil y por el sur hasta Talcahuano, Chile (Collette \& Nauen, 1983). La captura del Bonito se ha reducido, la FAO reporta para 1999, 3.187 toneladas; siendo los países con las mayores capturas México con 1.775 t y Perú con 948 t (Collette \& Nauen). Esto pone en manifiesto que la pesquería del Bonito va en declive y que a pesar de los años de explotación pesquera los antecedentes de su desarrollo temprano son escasos como para poder establecer planes de manejo de la especie en su medio natural o que genere antecedentes de base para su manipulación en sistemas de cultivos acuícolas. El éxito en la producción acuícola pasa por el conocimiento de las características morfofisiológicas y conductuales de la especie a cultivar, siendo vital el estudio de los primeros días de vida (Pezzato, 1997; Bota et al., 2010), ya que permite el correcto manejo de los recursos, la detección de alteraciones relacionadas con los factores ambientales en las incubadoras que puedan llevar malformaciones de las larvas y consecuentemente una menor productividad (Alves \& Moura, 1992). La bibliografía existente sobre aspectos biológicos reproductivos de Sarda ch. chiliensis es escasa, por ello se hace fundamental aportar nuevos datos, por el interés que ha adquirido su desarrollo a través de la piscicultura, de ahí la necesidad de caracterizar su desarollo embrionario y larval.

\section{MATERIAL Y MÉTODO}

La investigación se realizó en el Centro de Estudios Marinos y Limnológicas de la Universidad de Tarapacá, ubicada en el sector costero al sur de la ciudad de Arica, en la playa "La Capilla" (Latitud 18.5167 S, y Longitud: 70.3333 W), de la Región de Arica y Parinacota, donde a partir del día 24 de enero de 2013, se recolectaron huevos con una red planctónica de $300 \mu \mathrm{m}$ de malla del tanque de cultivo de 60 cubos de adultos reproductivos de bonito pertenecientes al proyecto "Desarrollo e implementación de las tecnologías de acondicionamiento y reproducción de peces pelágicos: bonito (Sarda chiliensis) y dorado (Seriola lalandi)", financiado por el Fondo de Fomento al Desarrollo Científico y Tecnológico de CONICYT. Dichos huevos se separaron por periodos de desarrollo y se distribuyeron en tres acuarios con aireación continua. Dos de los acuarios tuvieron una capacidad de $1,5 \mathrm{~L}$ y un tercero de $10 \mathrm{~L}$, los que se ubicaron dentro de una sala climatizada. Cuando los huevos alcanzaron el estado de larva, se alimentaron con rotíferos (Brachionus plicatilis), nauplios de artemia (Artemia fransciscana) y para los periodos de mayor desarrollo se alimentaron con larvas de lisa (Mugil cephalus). El tiempo de muestreo correspondió a 45 días.
Definición de caracteres morfológicos de embriones y larvas. De cada grupo de estudio se tomaron en los primeros tres días, 10 muestras cada una hora al azar, lo que tiene relación con lo mencionado por Schultz, quién señala que son 3 días el periodo embrionario que presenta Sarda chiliensis. Una vez ocurrida la eclosión y tras obtener las larvas, el periodo de muestreo se realizó cada dos, tres horas y una vez al día en la medida que los cambios se hicieron más lentos. Los huevos y larvas luego de ser caracterizados, fueron fotografiados y fijados en formalina al 5\% y alcohol al 30\%. La descripción fue realizada a través de un estereoscopio de Marca Japan Óptica Co., modelo XTL-2310 de 4,5x por 10x. Las fotografías se obtuvieron con una cámara digital de 16.1 Megapixeles de marca Samsung, modelo ST66 y del estereoscopio a través de la cámara para microscopia, modelo Moticam 1000 de 1,3 Megapixel. Simultáneamente se registraron la temperatura y $\mathrm{pH}$ a través del multiparámetro Hanna Instruments Waterproof pH Testers, modelo HI 98127.

Morfometría de los diferentes estadios de desarrollo. Para establecer la morfometría se midió la mayoría de los periodos de desarrollo identificados en la investigación. Las abreviaturas empleadas para los huevos fueron horas post-fecundación (HPF), diámetro del huevo horizontal (DHH) y vertical (DHV), longitud y alto del saco vitelino (LV y AV), longitud del rostro (LR), medida entre el borde del hocico y el margen anterior del ojo; longitud y alto del ojo (LO y AO), medido a partir de las líneas media horizontal y vertical del ojo y diámetros de la gota oleosa (DGOH) horizontal y vertical (DGOV). El volumen del saco vitelino (Vsv) y de la gota oleosa (Vgo) en larvas se calcularon según fórmulas propuestas por Cetta \& Capuzzo (1982) y Aristizabal (2006), quienes consideran al saco vitelino como una elipse y a la gota oleosa como una esfera, donde L es el eje mayor de la elipse; $\mathrm{H}$ es el eje menor (vertical) de la elipse y $\mathrm{r}$ el radio de la esfera en milímetros. Para la determinación del volumen del saco vitelino (Vsv):

$$
\mathrm{Vsv}=\pi / 6 \mathrm{LH}^{2}
$$

Para la determinación del volumen de la gota oleosa (Vgo):

$$
\mathrm{Vgo}=4 / 3 \pi \mathrm{r}^{3}
$$

Muestreo. Se tomó como variable de diseño la longitud total

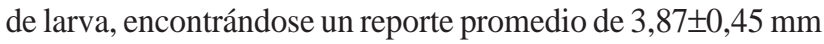
para Sarda sarda (Ortega \& De la Gándara, 2007), lográndose con estos valores estimar una varianza y un error relativo en función de esta media. El criterio fue de muestreo aleatorio simple, con un $10 \%$ de error y una confiabilidad del $95 \%$, encontrándose un tamaño muestral final suficiente de $n=6$. 
Descripción e Inferencia. Para determinar si existieron diferencias significativas entre las mediciones morfométricas para embriones se procedió a realizar una estadística descriptiva y comparativa para dos grupos de desarrollo (embriones en neurulación y embriones en metamería), donde previamente se realizó un análisis de normalidad, cuando ésta fue rechazada la comparación se realizó mediante test de medianas, en caso contrario se recurrió a la prueba t, ambos con una confiabilidad del 95\%. También se requirió de técnicas multivariadas de componentes principales, utilizando la lógica de retener componentes en función del gráfico de sedimentación. En larvas se realizaron análisis multivariados debido al número elevado de variables, recurriendo a la técnica de componentes principales ocupando como criterio de medición del número de componentes a retener: el de gráfico de sedimentación. Para longitud de larvas se buscó un modelo que relacionara este parámetro con el tiempo, ajustando una curva, encontrando los estadísticos de bondad del ajuste, pesando el modelo mediante un ANOVA y determinando la significancia individual de cada parámetro de la ecuación de regresión encontrada en la estadística t. Para todos los análisis requeridos, se utilizó el software estadístico SPSS 16.0. Para determinar si la temperatura del agua del cultivo pudo incidir en los resultados, se procedió a plotear las temperaturas en función del tiempo, generando así una gráfica temperatura-tiempo.

Definición del porcentaje de sobrevivencia de huevos y de eclosión larval. Para la definición de los porcentajes de sobrevivencia de huevos, se estableció el conteo de aquellos viables que antes de eclosionar, flotaban en superficie, y la sobrevivencia larval como el cuociente entre las larvas eclosionadas y viables y huevos sobrevivientes. Para esto, se estableció un grupo de estudio y dos réplicas. Se montó un sistema cerrado en un acuario de $30 \mathrm{~L}$ en un cuarto climatizado, en la que se introdujeron 6 recipientes de incubación con $250 \mathrm{~mL}$ de agua de mar filtrada, cada uno con 35 huevos fertilizados en el mismo estadio de desarrollo de 45 HPF. Las temperaturas y $\mathrm{pH}$ de incubación fueron para el grupo de estudio, de $23,142 \pm 0,051^{\circ} \mathrm{C}$ y $\mathrm{pH} 8,3 \pm 0$, para la réplica 1 fueron de; $23,933 \pm 0,446^{\circ} \mathrm{C} \mathrm{y} \mathrm{pH}$ $8,275 \pm 0,087$ y finalmente para la réplica 2 fueron de $24,833 \pm 0,103^{\circ} \mathrm{C}$ y $\mathrm{pH} 8,3 \pm 0,063$. Los porcentajes de sobrevivencia de huevos y eclosión se calcularon mediante las siguientes fórmulas propuestas por Ottesen \& Bolla (1998), donde:

Porcentaje de sobrevivencia de huevos $=(\mathrm{n}$ de larvas eclosionadas/n total de huevos incubados) x 100 .

Porcentaje de eclosión $=(\mathrm{n}$ larvas viables $/ \mathrm{n}$ de sobrevivencia de huevos) x 100 .
Para determinar si el porcentaje de sobrevivencia y eclosión en los grupos de tratamiento presentaron diferencias significativas, se planteó la hipótesis nula de normalidad de datos, al rechazarse ésta, se implementó la comparación de medianas en base a criterio de grupos creados en base a temperaturas.

\section{RESULTADOS}

Desarrollo embrionario. Se encontró que los coeficientes de variabilidad son relativamente altos para variables como "alto cabeza", "alto ojo", "longitud de ojo" y "longitud de cabeza", cuando se compararon los embriones en neurulación y metamería, encontrándose diferencias estadísticas significativas $(\mathrm{p}<0,05)$ para dichas variables, reportándose en los demás casos la aceptación de la hipótesis nulas de igualdad de medias (Tablas I y II). El carácter "longitud de cabeza", al mostrar una distribución de variables no normal, se comparó entre estadios basándose en la prueba de medianas, rechazándose la hipótesis nula de igualdad, debido a diferencias estadísticas significativas $(\mathrm{p}<0,015)$. La metodología de componentes principales (Fig. 1A), en la que se englobó las variables morfométricas DHV, DHH, alto de cabeza, longitud de cabeza, DGOH, DGOV, AO y LO, arrojó dos componentes, el primero explica el $60 \%$ de la variabilidad y el segundo el 15\%, es decir ambos explican el $85 \%$ acumulado.

Desarrollo larval. Las larvas recién eclosionadas midieron en promedio $4,116 \pm 0,529 \mathrm{~mm}$ y el estadio pre-juvenil alcanzó una longitud total de $16 \mathrm{~mm}$ (Fig. 1B). Se elaboró un modelo para las variables "longitud total de larvas/horas de vida" (Fig. 1C), donde el estadístico de bondad de ajuste indica una elevada capacidad de ajuste del modelo (Tabla III) que sirve de modo descriptivo-explicativo de orden cúbico $\left(\right.$ larvas $=4+0,003$ hora $+1,97 \mathrm{E}^{-0,7}$ hora $\left.^{3}\right)$, en donde los parámetros individuales fueron todos significativos estadísticamente. El ANOVA para la regresión cúbica computa el mismo record, lo cual fue corroborado por los estadísticos de bondad del ajuste, todos superiores al 93\%, y el error de estimación es bajo (Tabla IV). Además se realizó una matriz de componentes (Tabla V) con las mediciones obtenidas de las larvas, las que están altamente correlacionadas, ya que la técnica de principales arrojó una sola componente como combinación lineal de las variables, entendiéndose que el estado natural de ellas es irreductible, es decir, no existe ganancia de información si se quieren fraccionar dichas variables. El conocer estos descriptivos permite sugerir que en estudios posteriores se evalúe aquellos que señalan cambios significativos en el desarrollo ontogénico, que quedarían remitidos a: "alto de cabeza" y 
Tabla I. Descriptivos para embriones en neurulación y metamería.

\begin{tabular}{lcccccccc}
\hline Variable & $\begin{array}{c}\text { DHV } \\
(\mathbf{m m})\end{array}$ & $\begin{array}{c}\text { DHH } \\
(\mathbf{m m})\end{array}$ & $\begin{array}{c}\text { Alto } \\
\text { Cabeza }\end{array}$ & $\begin{array}{c}\text { Longitud } \\
\text { Cabeza }\end{array}$ & $\begin{array}{c}\text { Alto gota } \\
\text { oleosa }\end{array}$ & $\begin{array}{c}\text { Longitud } \\
\text { gota oleosa }\end{array}$ & $\begin{array}{c}\text { Alto } \\
\text { ojo }\end{array}$ & $\begin{array}{c}\text { Longitud } \\
\text { ojo }\end{array}$ \\
\hline Media (X) & 1,469 & 1,622 & 0,280 & 0,486 & 0,283 & 0,331 & 0,183 & 0,322 \\
Error estándar & 0,471 & 0,463 & 0,209 & 0,212 & 0,094 & 0,099 & 0,113 & 0,155 \\
Desviación estándar & 0,240 & 0,236 & 0,107 & 0,108 & 0,048 & 0,050 & 0,058 & 0,079 \\
Coeficiente & 16,347 & 14,561 & 38,066 & 22,242 & 16,877 & 15,220 & 31,561 & 24,575 \\
\hline
\end{tabular}

Tabla II. Prueba t para los embriones en neurulación y metamería.

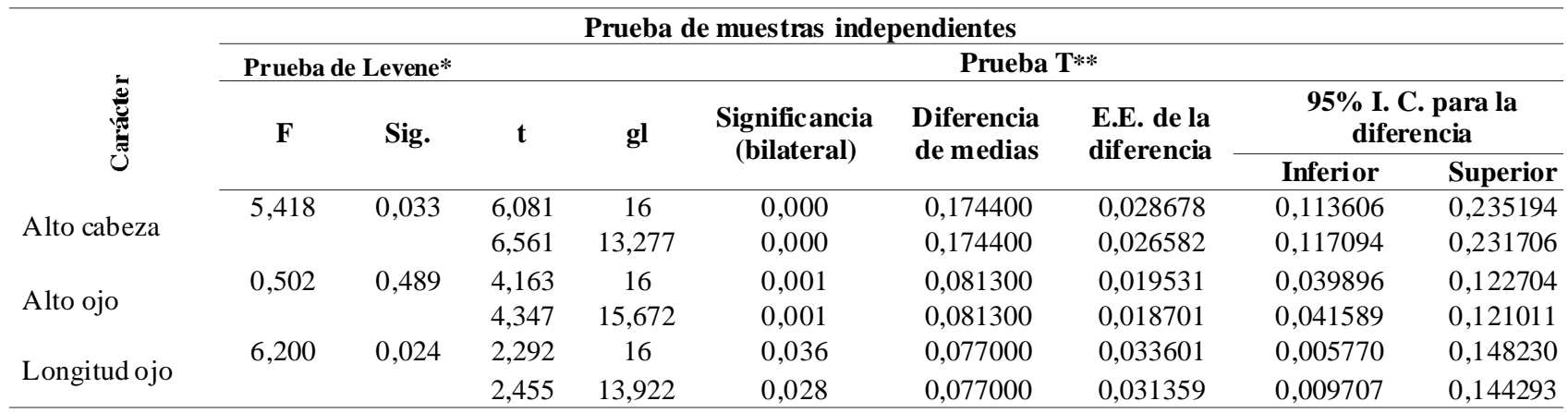

*=Prueba de Levene para la igualdad de varianzas; **= Prueba T para la igualdad de medias.

A

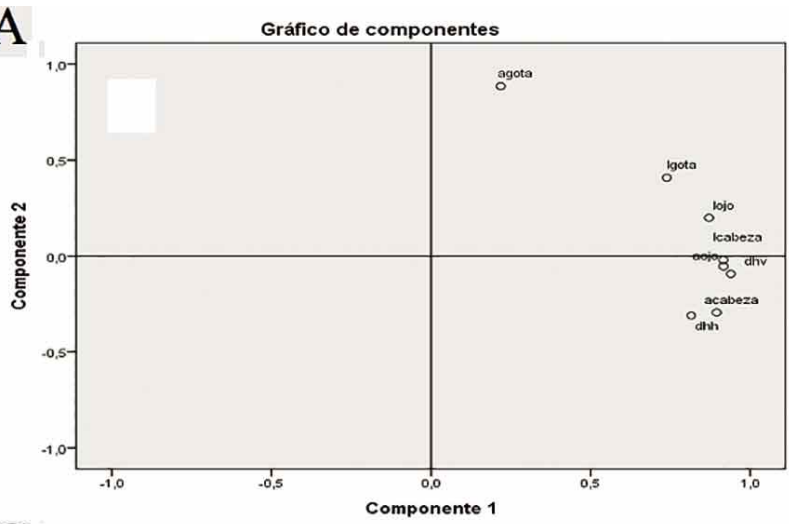

$\mathrm{C}$

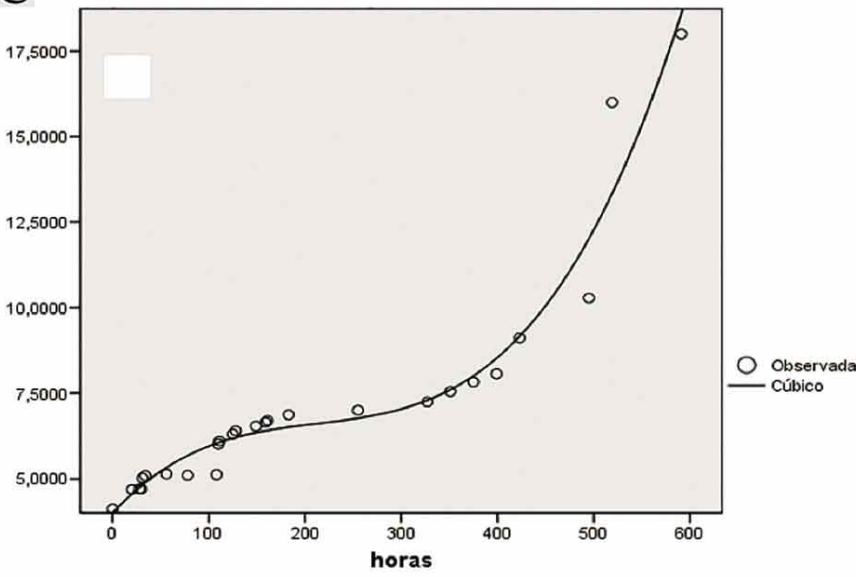

B Crecimiento en larvas de Sarda ch. chiliensis

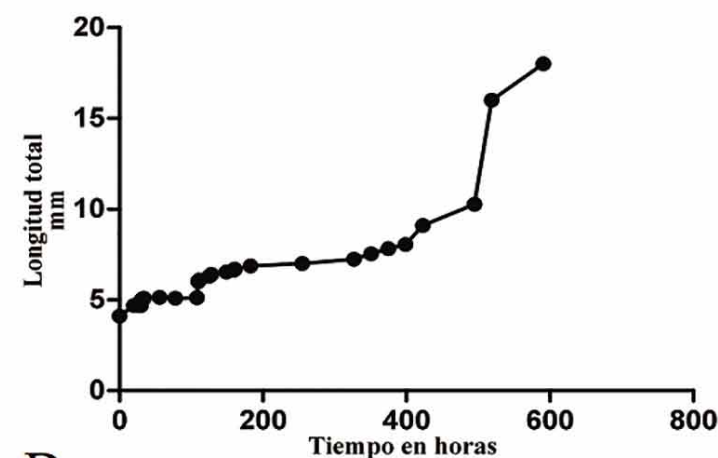

D

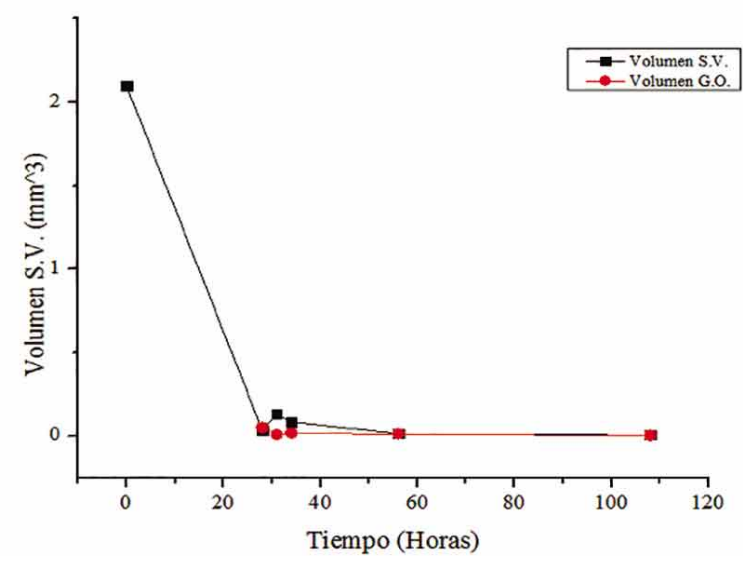

Fig. 1. En A) se muestra la gráfica de componentes para las variables morfométricas de embriones. En B) la longitud total de larvas de Sarda ch. chiliensis, según sus horas de vida. En C); longitud total de larvas de Sarda ch. chiliensis, según modelo cúbico de crecimiento. En D) el volumen del saco vitelino y volumen de la gota oleosa, según las horas de desarrollo larval. 
"longitud de cabeza" (c.v.: 22,242), "alto de ojo" (c.v.: 31,561) y "longitud de ojo" (c.v.: 24,575). La determinación de los volúmenes del saco vitelino y de la gota oleosa y de su reducción en el tiempo del desarrollo larval, permite estimar relaciones con la aparición de caracteres y conductas relacionadas al cambio de alimentación (Fig. 1D).

Con relación a definir el porcentaje de sobrevivencia y eclosión larval. El promedio total de sobrevivencia de huevos de los grupos analizados fue de $86,316 \pm 9,808 \%$, observándose que el menor porcentaje fue de un $82,282 \%$ a una temperatura de $24,833^{\circ} \mathrm{C}$ con un $\mathrm{pH}$ de 8,3 y el mayor porcentaje fue de $88,571 \%$ a una temperatura de $23,933^{\circ} \mathrm{C}$ con un pH de 8,275 (Tabla VI). En tanto el promedio total del porcentaje de eclosión fue más elevado, de un $89,596 \pm 11,683 \%$, donde el menor porcentaje de eclosión fue de $85,107 \%$ lo que coincide con el grupo de estudio que presentó el menor porcentaje de sobrevivencia, ambos registraron la temperatura más alta; en tanto que el mayor porcentaje de eclosión fue de un $92,389 \%$ con la temperatura más baja de los tres grupos, de $23,142^{\circ} \mathrm{C}$ y un $\mathrm{pH}$ de 8,3 (Tabla VII).

Tabla III. Estadístico de bondad de ajuste del modelo "longitud total de larvas/horas de vida".

\begin{tabular}{cccc}
\hline \multicolumn{3}{c}{ Resumen del modelo } \\
\hline $\mathrm{R}$ & $\mathrm{R}$ cuadrado & $\mathrm{R}$ cuadrado corregida & Error típico de la estimación \\
\hline 0,976 & 0,953 & 0,946 & 0,753 \\
\hline
\end{tabular}

Tabla IV. Análisis de ANOVA para la regresión de la curva de crecimiento cúbico.

\begin{tabular}{cccccc}
\hline \multicolumn{7}{c}{ ANOVA } \\
\hline & Suma de cuadrados & gl & Media cuadrática & Sig. \\
\hline Regresión & 251,717 & 3 & 83,906 & 147,846 & $\mathbf{0 , 0 0 0}$ \\
Residual & 12,485 & 22 & 0,568 & \\
Total & 264,202 & 25 & La variable independiente es hora. \\
\hline \multicolumn{7}{c}{ Coeficientes } \\
\hline \multicolumn{7}{c}{ Sig. } \\
\hline \multicolumn{7}{c}{ Coeficientes estandarizados } \\
hora & Coeficientes no estandarizados & Beta & t \\
hora $* * 2$ & B & Error típico & 1,624 & 0,000 \\
hora $* * 3$ & 0,030 & 0,007 & $-3,782$ & 0,000 \\
$($ Constante $)$ & 0,000 & 0,000 & 3,203 & $-4,389$ & 0,000 \\
\hline
\end{tabular}

Tabla V. Matriz de componentes para las larvas de Sarda chiliensis chiliensis.

\begin{tabular}{lc}
\hline \multicolumn{2}{c}{ Matriz de componentes } \\
\hline & Componente \\
\hline Alto vitelo & $\mathbf{1}$ \\
Longitud del vitelo & 0,990 \\
Alto gota oleosa & 0,988 \\
Longitud gota oleosa & 0,998 \\
Radio de la gota oleosa & 0,974 \\
Volumen saco vitelino & 0,993 \\
Volumen gota oleosa & 0,977 \\
\hline
\end{tabular}

Tabla VI. Porcentaje de sobrevivencia de huevos de Sarda chiliensis chiliensis.

\begin{tabular}{cccc}
\hline Recipiente & Grupo de estudio & Réplica 1 & Réplica 2 \\
\hline 1 & 82,857 & 88,571 & 81,25 \\
2 & 100 & 91,429 & 100 \\
3 & 88,517 & 94,286 & 75 \\
4 & 82,857 & 88,571 & 93,75 \\
5 & 85,714 & 85,714 & 56,25 \\
6 & 88,571 & 82,857 & 87,5 \\
Promedio & 88,086 & 88,571 & 82,282 \\
Des viación & $\pm 6,367$ & $\pm 4,041$ & $\pm 15,520$ \\
Temperatura & $23,142^{\circ} \mathrm{C}$ & $23,933^{\circ} \mathrm{C}$ & $24,833^{\circ} \mathrm{C}$ \\
pH & 8,3 & 8,275 & 8,3 \\
\hline
\end{tabular}


Tabla VII. Porcentaje de eclosión de Sarda chiliensis chiliensis.

\begin{tabular}{cccc}
\hline Recipiente & Grupo de estudio & Réplica 1 & Réplica 2 \\
\hline 1 & 100 & 96,774 & 92,308 \\
2 & 97,143 & 91,176 & 50 \\
3 & 90,323 & 87,878 & 75 \\
4 & 93,103 & 87,5 & 93,333 \\
5 & 86,667 & 87,879 & 100 \\
6 & 87,097 & 96,552 & 100 \\
Promedio & 92,389 & 91,293 & 85,107 \\
Desviación & $\pm 5,405$ & $\pm 4,368$ & $\pm 19,482$ \\
Temperatura & $23,142^{\circ} \mathrm{C}$ & $23,933^{\circ} \mathrm{C}$ & $24,833^{\circ} \mathrm{C}$ \\
pH & 8,3 & 8,275 & 8,3 \\
\hline
\end{tabular}

\section{DISCUSIÓN}

Desarrollo embrionario. Los huevos vivos de Sarda ch. chiliensis son translúcidos, flotantes, del tipo telolecítico, y presentan una división meroblástica discoidal durante los primeros estadios del desarrollo, con dimensiones de $1,469 \pm 0,240 \mathrm{~mm}$ de DHV y $1,622 \pm 0,236 \mathrm{~mm}$ de DHH, con 1 a 6 gotas oleosas (Fig. 2A), las que a partir de las 57 horas de desarrollo tendieron a fusionarse en una sola, probablemente para facilitar la flotación. El tiempo transcurrido desde la fecundación hasta la eclosión fue de 71,833 horas, lo que concuerda con Schultz, que son 3 días el periodo embrionario que presenta Sarda chiliensis. Los registros obtenidos para Sarda sarda (bonito del Atlántico), en cautiverio fueron de huevos con un diámetro de $1,048 \pm 0,103 \mathrm{~mm}$, cuyo número de gotas oleosas osciló entre 1 y 8 , los que eclosionaron tras 48 horas de incubación a $21^{\circ} \mathrm{C}$, su desa- rrollo embrionario fue más rápido, con las primeras divisiones entre las 01:30 h - 01:45 h tras la fecundación, el estado de mórula se registró tras unas cinco horas, a las 24 horas la formación del embrión y horas más tarde la segmentación (Ortega \& de la Gándara). En tanto, en el Monterey Bay Aquarium, de Monterey, California, EEUU; McFarlane et al. (2000), señalan que Sarda ch. lineolata, también en cautiverio, el diámetro de los huevos recuperados fue de 1,58 $\mathrm{mm}+/-0,05$ y que comenzaron a nacer dentro de las 48 horas del desove, donde el tamaño de huevos fue similar a los de poblaciones salvajes del bonito del Pacífico (1,52 a $1,68 \mathrm{~mm}$ ) señalado por Ambrose (1996). El periodo de eclosión de Sarda ch. lineolata coincide con Sarda sarda, no así con Sarda ch. chiliensis, que le tomó un día más para eclosionar. Según lo observado, Sarda ch. chiliensis con re-

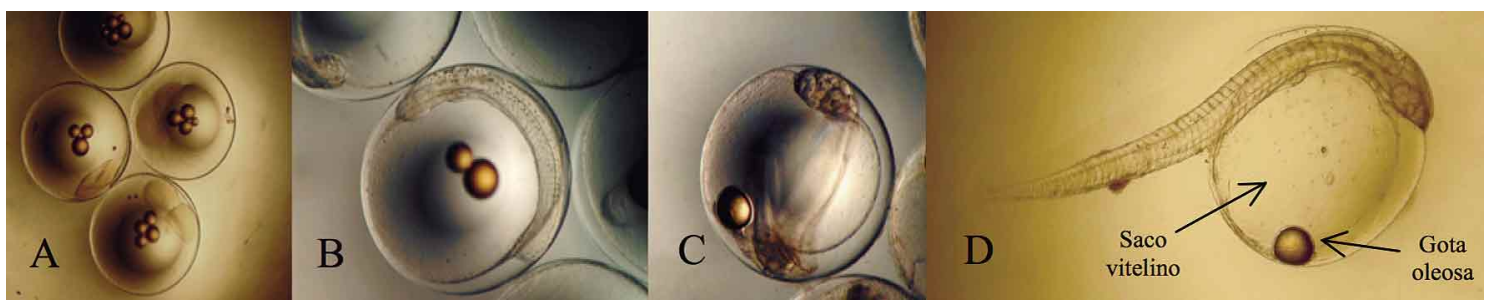

Fig. 2. Se observan diferentes estadios de desarrollo embrionario de Sarda chiliensis chiliensis En A): Las primeras divisiones mitóticas y distinto número de gotas oleosas (1 a 6). En B): Neurulación. En C): Metamería. En D) Se observa una larva recién eclosionada con una sola gota oleosa (flecha) al extremo posterior del saco vitelino (flecha), aún no termina y la presencia de cromatóforos que le dan una tenue tonalidad amarillenta.

Tabla VIII. Se presentan los tamaños de huevos y las diferencias entre cada especie y Sarda chiliensis chiliensis en milímetros.

\begin{tabular}{lcc}
\hline \multicolumn{1}{c}{ Especie } & $\begin{array}{c}\text { Tamaño de huevo } \\
\text { en } \mathbf{~ m m}\end{array}$ & $\begin{array}{c}\text { Diferencias } \\
\text { en } \mathbf{~ m m}\end{array}$ \\
\hline Sarda sarda & 1,048 & 0,574 \\
Sarda chiliensis lineolata & 1,58 & 0,042 \\
Sarda chilie nsis chiliensis & 1,622 & -- \\
\hline
\end{tabular}


lación al tamaño de huevo, tiene un rango intermedio entre ambas especies, siendo más cercana a Sarda ch. lineolata que a Sarda sarda (Tabla VIII). Con relación a los huevos fecundados de Sarda ch. chiliensis, a los 70 minutos $(1,167$ horas) se observó la presencia de un primer blastómero; a los 80 minutos ( 1,33 horas) la primera división y la formación de un huevo con 4 blastómeros a las 2 horas, lo que coincide con Sarda sarda. Se observó una mórula de 16 células a los 160 minutos (2,667 horas) y una mórula avanzada a los 320 min (5,333 horas), que también coincide con lo observado por Ortega \& De la Gándara, ya que señalan un periodo de 5 horas, también hay similitud con el periodo en que aparece el esbozo del embrión en Sarda sarda que es de 24 horas y para Sarda ch. chiliensis es de 28 horas. Además se puede observar que el comienzo de la somitogenésis en Sarda ch. chiliensis se da antes de que el blastodermo cubra totalmente la célula vitelina, lo que ocurre aproximadamente a las $36 \mathrm{HPF}$, esta situación ya ha sido descrito en el desarrollo de otras especies de teleósteos (Botta et al., 2010).

Con respecto a los caracteres morfométricos, la diferencia entre estadios embrionarios está dada por el desarrollo de la región cefálica donde se presentaron los más altos coeficientes de variabilidad en "alto cabeza", "alto ojo", "longitud de ojo" y "longitud de cabeza", no así para el resto de los caracteres medidos donde la variabilidad fue bastante similar. Esto señala que los cambios en los diferentes estadios estarían dados principalmente por estas cuatro variables morfométricas, y no sería necesario pesquisar las restantes. Esta información es fundamental ya que permitiría en estudios consecutivos optimizar el tiempo de muestreo y analizar un mayor número de muestras.

Desarrollo larval. La larva recién eclosionada de Sarda ch. chiliensis, se caracteriza por ser completamente traslúcida al estereoscopio, presenta unos pequeños cromatóforos puntiformes casi imperceptibles y sin un patrón aparente a lo largo de la larva, que le dan una sutil tonalidad amarillenta (Fig. 2D), que tiende a no notarse en la medida que la larva continua con su desarrollo, esto concuerda con lo observado por Barnhart (1927) para las larvas de Sarda ch. lineolata, obtenidas de huevos recogidos de La Jolla, California, posteriormente incubados en laboratorio. Dicho autor, acredita que las larvas recién eclosionadas presentan unos pocos cromatóforos de color amarillo, por el otro lado Sanzo (1932) y Vodyanitsky (1936) para Sarda sarda, muestran que, además de la pigmentación amarilla en las larvas, hay melanóforos en los huevos justo antes de la eclosión, situación que no se observó en Sarda ch. chiliensis, ya que ni en embriones ni en periodos tempranos de desarrollo larval se observaron melanóforos. Existe además, un lento crecimiento larval entre los 2 y 4 días después de la eclosión (Fig. 1B, etapa de neurulación), coincidiendo con la apertu- ra de la boca, el agotamiento del saco vitelino y gota oleosa, e inicio de la alimentación externa (2,333 días) con aún un insipiente desarrollo de la dentadura, ya recién a las 108 horas de vida se aprecia bien desarrollada (4,5 días). Comparándolo con Sarda sarda, las larvas recién nacidas miden $3,87 \pm 0,45 \mathrm{~mm}$, siendo su crecimiento larvario rápido y donde la metamorfosis se completa hacia el día 40 de vida (Ortega \& Dela Gándara, 2007), en tanto que las larvas de Sarda ch. chiliensis recién eclosionadas midieron en promedio $4,116 \pm 0,529 \mathrm{~mm}$, siendo ligeramente más largas. Por su parte McFarlane et al. agregan que para las larvas del primer estadio de Sarda ch. lineolata el diámetro de ojo se encuentra en un rango entre $0,34-0,36 \mathrm{~mm}$; asumiendo que el rango inferior es para las larvas recién eclosionadas, habría una similitud con Sarda ch. chiliensis quién registró en larvas recién eclosionadas un promedio de alto de ojo de 0,283 $\mathrm{mm}$ y un promedio de longitud de $0,346 \mathrm{~mm}$, siendo éste último concordante con lo señalado por McFarlane, en tanto que en Sarda sarda, los datos están faltantes.

Otra de las diferencias observadas con respecto a las especies citadas, es la única presencia de una gota oleosa en estado larval, que se presenta ya en las últimas fases de desarrollo embrionario hasta su total reabsorción a 89 horas de vida, donde ya no se observa (3,708 días), siendo éste periodo, la extinción de la última fuente endógena de alimentación. Con respecto a la alimentación, cuya disponibilidad y abundancia, es crucial para la sobrevivencia larval, Houde (1974), señala que el tiempo desde la eclosión hasta la pigmentación de los ojos representa el tiempo en el cual las larvas pueden nutrirse sólo de sus reservas vitelinas. Esta situación se observó a partir de las 53 horas de vida (2,208 días), donde las larvas tenían el ojo completamente pigmentado y comenzaron a predar sobre rotíferos (paulatinamente los ojos comenzaron a pigmentarse a partir de las 34 horas), aunque aún conservaban un escaso saco vitelino rodeando la única gota oleosa, de hecho en la gráfica del volumen del saco vitelino versus el tiempo de vida larval (Fig. 1D), se puede observar que entre las 28 a 30 horas ocurrió un fuerte consumo del vitelo, lo que podría ser gatillante de la pigmentación del ojo. La gota oleosa presentó la misma tendencia que el saco vitelino, donde aproximadamente a las 28 horas se observó una fuerte reducción, siendo ésta, la última en consumirse. Al momento de comenzar su alimentación exógena, el volumen del saco y la gota, fueron mínimos y se mantuvieron así hasta aproximadamente las 90 horas de vida, por lo que se puede inferir, que a partir de las 53 horas, el principal aporte energético lo pasó a constituir las fuentes exógenas de alimento. Si sería interesante conocer el comportamiento de la curva entre las 0 y 28 horas de vida larval, siendo necesario disgregar la información a intervalos regulares y más cortos de tiempo a fin de intencionar la búsqueda de un polinomio de grado n. Esta situación re- 
salta la importancia del desarrollo ocular en la captura de sus presas, lo que tiene directa relación con su comportamiento altamente depredador observado. Este escenario coincide con lo observado para Sarda sarda, ya que las larvas comenzaron a alimentarse de rotífero a los dos días de vida (Ortega \& De la Gándara), en tanto que las larvas de Sarda ch. lineolata comenzaron a predar sobre rotíferos y copépodos en el estadio L2 que comienza en el día 4, cuando las larvas tienen 5,5 mm, de acuerdo a McFarlane et al. Estos acontecimientos señalan otra vez la mayor cercanía en desarrollo y comportamiento que tienen Sarda ch. chiliensis con Sarda sarda.

Para Sarda ch. chiliensis, muchos cambios ontogenéticos observados estaban relacionadas con un comportamiento sumamente depredador, que involucraron un rápido desarrollo de la cabeza, ojos y boca, y además un creciente carácter esquivo de las fuentes de alimentos ofertados, si bien las larvas de 53 horas inicialmente predaban sobre algas como rotíferos (Brachionus plicatilis), en la medida que el desarrollo bucal se hizo más notorio, dejaron de preferir este alimento y empezaron a predar sobre los mismos de menor tamaño, lo que se identificó aproximadamente a las 152 horas de vida (6,333 días de vida), la misma situación se observó cuando se les ofertó nauplios de artemias (Artemia franciscana), preferían desarrollar conductas caníbales, antes de consumir estos nauplios; ambos a pesar de ser alimentos ampliamente señalados por la literatura para hatchery de peces marinos, no resulta ser la mejor opción, y la alimentación de éstos deberá ser una variable de importancia a abordar en próximos estudios. Con respecto a Sarda ch. lineolata, los hechos más destacables son la presencia de dos eventos metamórficos discretos durante el desarrollo (Youson, 1988) que también se observan en Sarda sarda, los que tendrían como elemento común la diferenciación de la primera aleta dorsal y caudal. En Sarda ch. lineolata, la primera metamorfosis se caracteriza por un rápido crecimiento, y el desarrollo de la musculatura del tronco, aletas huesudas y la mandíbula, además hay una mejora global en la capacidad del aparato locomotor, los que se producen en un periodo menor a 12 horas en un individuo dado a $26^{\circ} \mathrm{C}$, en tanto que la segunda metamorfosis más lenta, terminaría de ocurrir en estado juvenil, donde adquiriría la forma propia de un adulto (McFarlane op cit.). Para Sarda sarda, la metamorfosis comienza entre los 12 a 15 días de vida y se completa hacia el día 40 de cuando la larva ya es juvenil (Ortega \& De la Gándara), a una temperatura promedio de $21^{\circ} \mathrm{C}$; dichos autores definen este estadio a partir de $\operatorname{los} 20$ a 25 días de vida.

Para Sarda ch. chiliensis, comenzar la primera metamorfosis, le tomó más tiempo, ya que esta diferenciación de aletas dorsal y caudal, se registró casi a los 22 días a una temperatura promedio de $23,75^{\circ} \mathrm{C}$. Además se pudo observar en Sarda ch. chiliensis, que la formación de escamas comenzó en la región cefálica y opercular en conjunto con la diferenciación de las aletas y retracción de la aleta embrionaria, con la pigmentación de los radios sobretodo de la primera aleta dorsal y aleta caudal, pero aún no tiene la forma corporal de un adulto, siendo gran parte del cuerpo aun traslúcido, por lo que correspondería a un periodo previo, denominado prejuvenil correspondiente a las 519 horas de vida (de 21,625 días de vida). Esto señalaría que para Sarda ch. chiliensis entre los 21 y 22 días de vida larval estarían bajo la primera metamorfosis que le permitiría adquirir la forma de un adulto, siendo la diferenciación de la primera aleta dorsal, el inicio del estadio de prejuvenil. En tanto que el periodo juvenil correspondería ya a la presencia de una escamación casi completa del cuerpo, la adopción de una forma fusiforme del cuerpo típica del adulto, la presencia de las características rayas oscuras oblicuas en el dorso de cuerpo que se extienden hacia los flancos del cuerpo que es algo comprimido, las espínulas, la línea lateral definida a lo largo del cuerpo, observándose además la presencia de una cabeza puntiaguda, cónica con una boca de gran tamaño donde se aprecian los grandes dientes cónicos, lo que ocurre después de las 600 horas (25 días).

Esto podría permitir inferir que aproximadamente a partir de las 495 horas (20,625 días) se daría inicio a la primera metamorfosis y de una segunda metamorfosis para dar juveniles a partir de las 591 horas (24,625 días). Con relación al establecimiento de un modelo de crecimiento (Fig. 1C), reafirman lo señalado anteriormente, donde se observan después de la eclosión tres grandes estadios, el larval, prejuvenil y juvenil. Si bien no se pudo lograr la sobrevivencia de juveniles, ésta se debió principalmente por que el alimento ofertado no fue el requerido, muriendo los individuos de inanición más que por condiciones físicasquímicas inadecuadas, ya que al realizar el cruce entre las longitudes totales larvales de los grupos de estudio y de las temperaturas y pH sometidos, el análisis arrojó independencia entre las variables, es decir, estas condiciones no incidieron en el desarrollo de la especie. Además que de los grupos de estudio, se obtuvieron los mismos morfos, no existiendo diferencias significativas entre éstos.

Con relación a definir el porcentaje de sobrevivencia y eclosión larval. Los antecedentes señalan que la temperatura controla el tiempo de incubación de los huevos y la supervivencia de embriones y larvas; cuando los huevos son incubados fuera de un intervalo óptimo, el desarrollo embrionario puede acelerarse o retardarse, produciendo embriones y larvas deformes, bajos porcentajes de eclosión y elevada mortalidad de larvas eclosionadas (Abdo-de la Parra et al., 2012). Si bien no hay información acerca de las 
condiciones óptimas para el desarrollo embrionario de Sarda ch. chiliensis en cautiverio, existen antecedentes de éxito para su congénere del océano Atlántico Sarda sarda. De acuerdo a McFarlane et al., la temperatura más adecuada para el éxito del cultivo del bonito está entre los $22-26^{\circ} \mathrm{C}$, ya que a temperaturas más altas hay un desarrollo más rápido con mortalidades más altas y a temperaturas más bajas, un desarrollo más lento y mortalidades inferiores; dentro de dicho rango citado de temperatura, se encuentra la actividad de desove observada en las poblaciones silvestres de Bonito del Pacífico de la Costa de California (Collette \& Nauen; Moser et al., 1993; McFarlane et al.) y en este rango de temperaturas se desarrolló el cultivo de Sarda ch. chiliensis para la caracterización morfológica y morfométrica $\left(23,75 \pm 1,450^{\circ} \mathrm{C}\right)$ como así la experiencia de sobrevivencia de huevos y eclosión de larvas, con una temperatura promedio de $23,797 \pm 0,697^{\circ} \mathrm{C}$, no existiendo diferencias significativas entre grupos. El promedio total de sobrevivencia de huevos fue de 86,316\% y el de eclosión más elevado, de un 89,596\%. En el Monterey Bay Aquarium, de Monterey, California, EEUU, McFarlane (op. cit.), señalan que Sarda ch. lineolata en cautiverio un $75 \%$ de los huevos fertilizados sobrevivieron a la eclosión. En tanto que Ortega \& De la Gándara, señalaron una tasa de eclosión de alrededor del $60 \%$ para Sarda sarda. De acuerdo a las temperaturas ópti- mas señaladas por McFarlane et al., las registradas para la determinación de los porcentajes de sobrevivencia y eclosión están más cercanas al rango inferior sugerido por dichos autores, lo que podría explicar los tiempos más largos en alcanzar cada estadio y los porcentajes altos en sobrevivencia y eclosión, por lo que en el cultivo de Sarda ch. chiliensis, se debiera esperar buenos resultados, si se logran mantener todas las variables bajo control.

Si bien Sarda ch. chiliensis, comparte características morfológicas y morfométricas con Sarda ch. lineolata y Sarda sarda; tiene más parentesco con esta última. De la comparación de los estadios morfológicos y morfométricas señalan que hay una pequeña diferencia en tiempos de desarrollo, más no en los eventos biológicos que se manifiestan en dichas especies. Esto permite inferir que si es factible el éxito de un hatchery de cultivo de Sarda ch. chilensis en sistemas de recirculación en la zona Norte de Chile, primero porque se logró obtener todos los estadios de desarrollo permitiendo establecer estadios de desarrollo ontológico para la especie, segundo porque los porcentajes de sobrevivencia y eclosión fueron los más altos con respecto a las especies citadas, y tercero dada la similitud de Sarda ch. chiliensis con Sarda sarda y de los antecedentes de respuestas exitosas del cultivo obtenidas en esta última.

MIRANDA, L.; VILAXA, A.; ÁVILA R. \& RODRÍGUEZ, M. Characterization of the stages in the cycle of embryo development Sarda chiliensis chiliensis (Alva, 1987) in an aquatic re-circulation system (SAR). Int. J. Morphol., 32(4):1492-1501, 2014.

SUMMARY: Aquatic re-circulation systems (SAR) are being used more and more in agriculture, especially in the north of Chile where the environment conditions are suitable for breeding in SAR (aquatic re-circulation system) of species such as Bonito (Sarda chiliensis chiliensis), whose embryo or larvae origins are unknown, which complicates the evaluation of a technical and economical possibility of developing its breeding in SAR. Due to this, bases for its breeding were determined in January 2013, for 45 days. The characterized embryo periods were 31 in 71, 83 hours, defined in 5 stages: Morula, Blastula, Gastrula, Neurulation and Metamery. From the larvae development 35 periods in 519 hours were observed, a pre-juvenile stage observed at 591 hours and a juvenile one. The eggs were translucent of a telolecitic type of a slow growing in relation to Sarda sarda and Sarda ch. lineolata, since it took them almost three days to hatch. The surviving and hatching rates were high $(86,316 \pm 9,808 \%$ and $89,596 \pm 11,683 \%$ respectively), so the conditions for their development would be appropriate. Meanwhile, the larvae presented more similarities with Sarda sarda in the first periods of development. The crucial period was between the 81 and 89 hours of life, when the oily drop was re-absorbed; besides, this was a distinguishing feature with the other two species because it was the only one that presented just one oily drop in the larvae period. The possibility of a Sarda ch. chiliensis hatchery is inferred, due to the similarities with Sarda sarda, and because of the success obtained in this breeding. From the morphometric characters, future monitoring is suggested, the ones that represent the most variables in the stages of development such as length and height of the head, and length and height of the eye.

KEY WORDS: Embryo development; Sarda chiliensis chiliensis.

\section{REFERENCIAS BIBLIOGRÁFICAS}

Abdo-de la Parra, M. I.; Martínez-Rodríguez, I. E.; GonzálezRodríguez, B.; Rodríguez-Ibarra, E.; Duncan, N. \& Hernández, C. Efecto de la temperatura y salinidad del agua en la incubación de huevos de botete diana Sphoeroides annulatus. Rev. Biol. Mar. Oceanogr., 47(1):147-53, 2012.
Alves, M. S. D. \& Moura, A. Estádios de desenvolvimento embrionário de curimatã-pioa Prochilodus affinis (Reinhardt, 1874) (Pisces, Prochilodontidae). In: Encontro anual de aquicultura de Minas Gerais, 10. Três Marias, CODEVASF, 1992. pp.61-71. 
Ambrose, D. A. Scombridae: mackerels and tunas. In: Moser, H. G. (Ed.). The early life stages of fishes in the California current region. Lawrence, Allen Press, 1996. pp.1270-85.

Aristizabal, E. O. Consumo de oxígeno, crecimiento y utilización del alimento durante el desarrollo larval del besugo Pagrus pagrus (Linné, 1758) (Pisces: Sparidae). Rev. Biol. Mar. Oceanogr., 41(2):209-20, 2006.

Barnhart, P. S. Pelagic fish eggs off La Jolla, California. Bull. Scripps Instn. Oceanogr. Tech. Ser. 1(8): 91-2, 1927.

Botta, P.; Sciara, A.; Arranz, S.; Murgas, L. D. S.; Pereira, G. J. M. \& Oberlender, G. Estudio del desarrollo embrionario del sábalo (Prochilodus lineatus). Arch. Med, Vet., 42(2):109-14, 2010.

Cetta, C. M.\& Capuzzo, J. M. Physiological and biochemical aspects of embryonic and larval development of the winter flounder Pseudopleuronectes americanus. Mar. Biol., 71(3):327-37, 1982.

Collette, B. B. \& Chao, L. N. Systematics and morphology of bonitos (Sarda) and their relatives (Scombridae, Sardini). Fish. Bull., 73(3):516-625, 1975.

Collette, B. B. \& Nauen, C. E. FAO Species Catalogue. Vol. 2. Scombrids of the world. An annotated and illustrated catalogue of Tunas, Mackerels, Bonitos and related species known to date. Rome, United Nations Development Programme, FAO Fisheries Synopsis, 1983.

Houde, E. D. Effects of temperature and delayed feeding on growth and survival of larvae of three species of subtropical marine fishes. Mar. Biol., 26(3):271-85, 1974.

McFarlane, M. B.; Cripe, D. J. \& Thompson, S. H. Larval growth and development of cultured Pacific bonito. J. Fish Biol., 57(1):134-44, 2000.

Moser, H. G.; Charter, R. L.; Smith, P. E.; Ambrose, D. A.; Sharter, S. R.; Meyer, C. A.; Sandknop, E. M. \& Watson, W. Distributional atlas of fish larvae and eggs in the California current region. Taxa with 1000 or more total larvae, 19511984. California, California Cooperative Oceanic Fisheries Investigations Atlas 31, 1993. pp.233.

Ortega, A. \& De la Gándara, F. Desarrollo embrionario y crecimiento larvario de bonito atlántico (Sarda sarda) nacido en cautividad. Pontevedra, XI Congreso Nacional de Acuicultura. Libro de Actas, 2007. pp.815-8.

Ottesen, O. H. \& Bolla, S. Combined effects of temperature and salinity on development and survival of Atlantic halibut larvae. Aquac. Int., 6(2):103-20, 1998.

Pezzato, L. E. O estabelecimento das exigências nutricionais das espécies cultivadas. Piracicaba, Simpósio sobre manejo e nutrição de peixes, 1997. pp.45-60.
Sanzo, L. Nuovo contributo alla conoscenza delle uova e primi stadi larvali negli Scomberoidi. Boll. Zool. 3:69-72, 1932.

Schultz, K. Ken Schultz's Field Guide to Saltwater Fish. Hoboken, Wiley, 2003. pp.276.

Vilaxa, A.; Silva, A. \& Pepe-Victoriano, R. Desarrollo e implementación de las tecnologías de acondicionamiento y reproducción de peces pelágicos: bonito (Sarda chiliensis) y dorado (Seriola lalandi) en la región de Arica-Parinacota. Temuco, II Congreso Nacional de Acuicultura - Libro de Resúmenes, 2009. pp.166.

Vodyanitsky, V. Nabliudenia nad pelagicheskimi iaitsami ryb Chernogo moria. Trudy Sevastopol. BioI. Sta., 5:3-43, 1936.

Yoshida, H. O. Synopsis of biological data on bonitos of the genus Sarda. Rome, United Nations Development Programme, FAO Fisheries Synopsis, 1980.

Youson, J. H. First metamorphosis. In: Hoar, W. S. \& Randall, D. J. (Eds.). Fish Physiology, Vol. 11B. San Diego, Academic Press, 1988. pp.135-96.

Dirección para Correspondencia:
Arnaldo Vilaxa Olcay
Universidad de Tarapacá
Arica
CHILE

Email: avilaxa@uta.cl

Recibido : 25-08-2014

Aceptado: 05-11-2014 